Check for updates

Cite this: Phys. Chem. Chem. Phys., 2020, 22, 10934

Received 5th March 2020

Accepted 23rd April 2020

DOI: $10.1039 / \mathrm{d} 0 \mathrm{cp} 01269 f$

rsc.li/pccp

\section{Decoding the molecular water structure at complex interfaces through surface-specific spectroscopy of the water bending mode $\uparrow$}

\author{
Takakazu Seki, (D) $\ddagger^{a}$ Chun-Chieh Yu, (D) $\ddagger^{a}$ Xiaoqing Yu, ${ }^{a}$ Tatsuhiko Ohto, \\ Shumei Sun, ${ }^{\text {acd }}$ Konrad Meister, ${ }^{\text {ae }}$ Ellen H. G. Backus, (D) ${ }^{\text {ad }}$ Mischa Bonn (D) *a and \\ Yuki Nagata (D)*a
}

\begin{abstract}
The structure of interfacial water determines atmospheric chemistry, wetting properties of materials, and protein folding. The challenge of investigating the properties of specific interfacial water molecules has frequently been confronted using surface-specific sum-frequency generation (SFG) vibrational spectroscopy using the $\mathrm{O}-\mathrm{H}$ stretch mode. While perfectly suited for the water-air interface, for complex interfaces, a potential complication arises from the contribution of hydroxyl or amine groups of non-water species present at the surface, such as surface hydroxyls on minerals, or $\mathrm{O}-\mathrm{H}$ and $\mathrm{N}-\mathrm{H}$ groups contained in proteins. Here, we present a protocol to extract the hydrogen bond strength selectively of interfacial water, through the water bending mode. The bending mode vibrational frequency distribution provides a new avenue for unveiling the hydrogen bonding structure of interfacial water at complex aqueous interfaces. We demonstrate this method for the water- $\mathrm{CaF}_{2}$ and waterprotein interfaces. For the former, we show that this method can indeed single out water $\mathrm{O}-\mathrm{H}$ groups from surface hydroxyls, and that with increasing $\mathrm{pH}$, the hydrogen-bonded network of interfacial water strengthens. Furthermore, we unveil enhanced hydrogen bonding of water, compared to bulk water, at the interface with human serum albumin proteins, a prototypical bio-interface.
\end{abstract}

\section{Introduction}

Aqueous interfaces play an important role in geology, biology, and chemistry. The hydration structure of proteins/lipids affects their biological functions, and the interaction of water with solid surfaces such as mineral and metal oxide surfaces determines surface chemistry and surface-catalytic reactions. Understanding the structure of water at interfaces is, therefore, of great interest. So far, surface-specific techniques such as scanning probe microscopy, X-ray spectroscopy, attenuated

\footnotetext{
${ }^{a}$ Max Planck Institute for Polymer Research, Ackermannweg 10, 55128 Mainz, Germany.E-mail: bonn@mpip-mainz.mpg.de,nagata@mpip-mainz.mpg.de

${ }^{b}$ Graduate School of Engineering Science, Osaka University, 1-3 Machikaneyama, Toyonaka, Osaka 560-8531, Japan

${ }^{c}$ Department of Physics, Applied Optics Beijing Area Major Laboratory, Beijing Normal University, Beijing 100875, China

${ }^{d}$ Department of Physical Chemistry, University of Vienna, Währinger Strasse 42, 1090 Vienna, Austria

${ }^{e}$ Department of Natural Sciences, University of Alaska Southeast, Juneau, AK 99801, USA

$\dagger$ Electronic supplementary information (ESI) available: Details of the SFG measurements, the fitting procedures, and the AIMD protocols. See DOI: 10.1039/d0cp01269f

\# These authors contributed equally.
}

total reflection-infrared (ATR-IR), and sum-frequency generation (SFG) spectroscopy have been used for investigating the molecular structure and dynamics of water at interfaces. ${ }^{1}$

Among them, vibrational SFG spectroscopy has been demonstrated to be a powerful technique to probe the interfacial water molecules at soft (biomolecule-water) ${ }^{2-7}$ and buried (solid-water) interfaces. ${ }^{8-17}$ SFG spectroscopy is active only in the centrosymmetry-broken region and therefore probes those molecular responses with a non-zero net transition dipole moment along the surface normal direction. As such, the molecules in the bulk and the molecular responses with their transition dipole moment being parallel to the surface are SFG-inactive. However, capturing the net contribution of the interfacial water in the $\mathrm{O}-\mathrm{H}$ stretch spectra is often highly challenging at the complex aqueous interfaces, because the contribution of the water $\mathrm{O}-\mathrm{H}$ stretch mode is spectrally indistinguishable from that of the $\mathrm{O}-\mathrm{H}$ groups chemisorbed onto the solid surface as well as the $\mathrm{O}-\mathrm{H}$ and $\mathrm{N}-\mathrm{H}$ groups of proteins. A small number of these $\mathrm{O}-\mathrm{H} / \mathrm{N}-\mathrm{H}$ groups can overwhelm the SFG signal, because the SFG signal of water arises from a very thin (sub-nm) water layer at the interfaces. These comparable contributions from the water $\mathrm{O}-\mathrm{H}$ groups and the other hydroxyl groups impede a clear molecular view of the interfacial water 
structure. For instance, a weakly hydrogen-bonded $\mathrm{O}-\mathrm{H}$ stretch peak in the SFG spectra at the water-silica interface has been conflictingly assigned to originating from interfacial water $^{18,19}$ and silanol groups on the silica surface. ${ }^{15}$ As such, an unambiguous determination of the interfacial water contribution is essential for unveiling the microscopic structure of water at interfaces. A second complication is the distortion of the SFG spectrum of the $\mathrm{O}-\mathrm{H}$ stretching mode due to intra- and intermolecular vibrational coupling effects. ${ }^{20}$

The net interfacial water contribution may be measured through the water bending mode, because the $\mathrm{H}-\mathrm{O}-\mathrm{H}$ bending mode originates solely from the $\mathrm{H}_{2} \mathrm{O}$ water molecules and has a spectrally distinguishable frequency from other $\mathrm{O}-\mathrm{H}$ bending modes. For example, the $\mathrm{H}-\mathrm{O}-\mathrm{H}$ bending mode frequency is $1590-1650 \mathrm{~cm}^{-1}$, which is significantly distinct from the bending mode frequency of hydroxyl groups attached on solid surfaces $\left(300-900 \mathrm{~cm}^{-1}\right) .{ }^{21}$ Since the first publication of the sum-frequency generation spectrum in the frequency region of the bending mode from the water-air interface, ${ }^{22}$ there have been debates about the surface-versus bulk-contribution to the signal. $^{23,24}$ We have recently demonstrated that the surface contribution to the overall spectrum can be readily extracted, paving the way for using the bending mode as a reporter for determining the interfacial water structure. ${ }^{24}$ The water bending mode may also be well-suited to study other complex interfaces such as protein interfaces. Although the strong amide I mode of proteins $\left(1620-1700 \mathrm{~cm}^{-1}\right)$ masks the $\mathrm{H}-\mathrm{O}-\mathrm{H}$ bending mode of water, the $\mathrm{H}-\mathrm{O}-\mathrm{D}$ bending mode of the isotopically diluted water $\left(1400-1450 \mathrm{~cm}^{-1}\right)$ can provide the information on the interfacial water.

A drawback to probe the water bending mode with SFG spectroscopy lies in its weak signal. The challenge in probing the bending mode SFG signal is further increased, when the bending mode signal is weakened by using the isotopically diluted water; if the bending mode lineshape is distorted by the intermolecular vibrational coupling of the bending modes, one has to obtain the characteristic frequency of the bending mode through the isotopic dilution of water. However, knowledge of the vibrational coupling on the bending mode in the SFG spectra is completely lacking. Thus, to establish a protocol to probe the interfacial water contribution through the bending mode SFG, one should first obtain the information on whether the bending mode SFG response is affected by vibrational coupling, by performing experiments with isotopic dilution.

Here, we demonstrate that the lineshape of the SFG bending mode signal is not changed upon the isotopic dilution from the SFG measurement at the water-air interface. We further compute the SFG spectra using the ab initio molecular dynamics (AIMD) simulations and confirm that the intermolecular coupling of the bending modes does not alter the lineshape of the bending mode. Subsequently, we obtain the net interfacial water contribution through the water bending mode at two aqueous interfaces. One is the water- $\mathrm{CaF}_{2}$ interface, where we decode the contribution of the interfacial water and find that the hydrogen bond strength is increased with increasing $\mathrm{pH}$. The other is the water-air interface in the presence of the human serum albumin (HSA) protein, where we use the $\mathrm{H}-\mathrm{O}-\mathrm{D}$ bending mode and discuss the strength of the water-HSA protein binding interaction. The successful determination of the interfacial hydrogen bond network through the SFG water bending mode manifests that probing the water bending mode in the SFG spectra is a promising tool for understanding the interfacial water structure.

\section{Results}

\section{Bending mode SFG spectra of water are unchanged upon isotopic dilution}

To examine how the lineshape of the bending mode varies upon the isotopic dilution of water, we measured the SFG intensity, $I^{(2)}(\omega) \sim\left|\chi_{\mathrm{NR}}^{(2)}+\chi_{\mathrm{R}}^{(2)}\right|^{2}$, in the $\mathrm{H}-\mathrm{O}-\mathrm{H}$ bending mode frequency region, at the water-air interface by varying the ratio of $\mathrm{H}_{2} \mathrm{O}$ and $\mathrm{D}_{2} \mathrm{O}$. $\chi_{\mathrm{R}}^{(2)}$ and $\chi_{\mathrm{NR}}^{(2)}$ denote the resonant and nonresonant contributions, respectively. The measured spectra are displayed in Fig. 1(a). The spectrum of neat $\mathrm{H}_{2} \mathrm{O}$ is consistent with those reported in previous studies. ${ }^{22,24-26}$ Upon isotopic dilution, the signal intensity decreased as expected. From the fit to $I^{(2)}(\omega)$ with a Lorentzian model, we obtained the peak frequency $\left(\omega_{\text {bend }}\right)$, half width at half maximum $\left(\Gamma_{\text {bend }}\right)$, and amplitude ( $\left.A_{\text {bend }}\right)$ of the bending mode of the $\mathrm{H}_{2} \mathrm{O}$ molecules donating two hydrogen bonds ${ }^{24-27}$ (double donors; see $\mathrm{ESI} \dagger$ ). The variations of $\omega_{\text {bend }}, \Gamma_{\text {bend }}$, and $A_{\text {bend }}$ are plotted in Fig. 1(b), (c), and (d), respectively.

Fig. 1(b) and (c) show that $\omega_{\text {bend }}$ is independent of the $\mathrm{H}_{2} \mathrm{O}$ concentration, while $\Gamma_{\text {bend }}$ shows a weak broadening with increasing $\mathrm{H}_{2} \mathrm{O}$ concentration. Fig. 1(d) shows that $A_{\text {bend }}$ is linearly proportional to the $\mathrm{H}_{2} \mathrm{O}$ concentration. Here, the $\mathrm{H}_{2} \mathrm{O}$ concentration was inferred using the equilibrium relation: ${ }^{28-30}$

$$
[\mathrm{HOD}]^{2} /\left[\mathrm{H}_{2} \mathrm{O}\right]\left[\mathrm{D}_{2} \mathrm{O}\right]=3.86 \text {. }
$$

The dotted lines in Fig. 1(b-d) show the response expected in the absence of vibrational coupling. Clearly, we find no
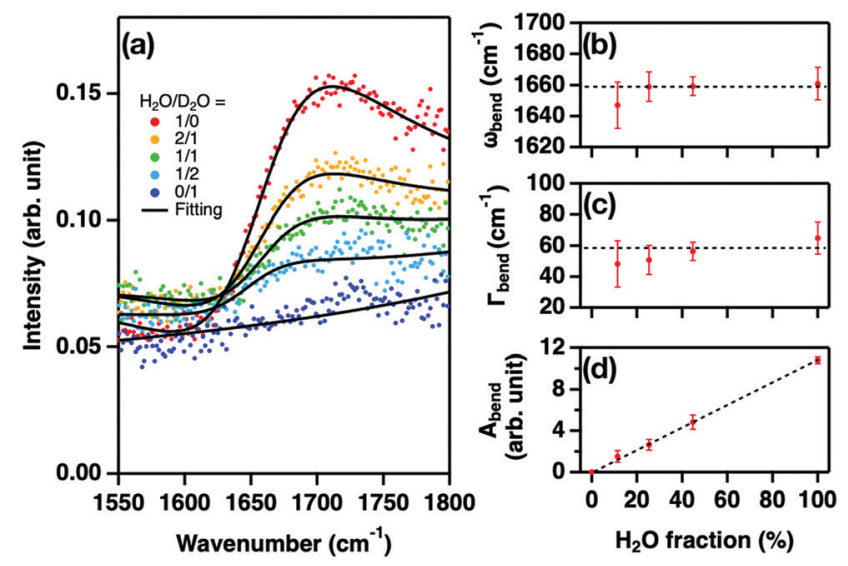

Fig. 1 SFG responses at the isotopically diluted water-air interface. (a) SFG intensity spectra, $I^{(2)}(\omega)$, of the $\mathrm{H}-\mathrm{O}-\mathrm{H}$ bending mode at the water-air interface with $1 / 0,2 / 1,1 / 1,1 / 2$, and $0 / 1 \mathrm{H}_{2} \mathrm{O} / \mathrm{D}_{2} \mathrm{O}$ mixtures. Solid lines are fits to the data. (b)Variations of $\omega_{\text {bend, }}$ (c) $\Gamma_{\text {bend, }}$ and (d) $A_{\text {bend. Dotted }}$ lines are the expected results in the absence of vibrational coupling. 


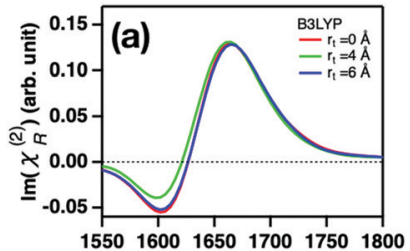

Wavenumber $\left(\mathrm{cm}^{-1}\right)$

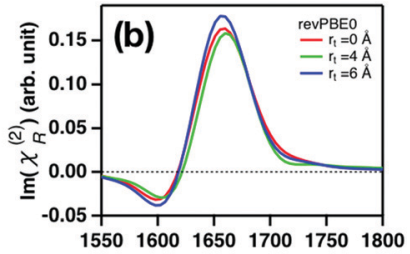

Wavenumber $\left(\mathrm{cm}^{-1}\right)$

Fig. 2 Simulated $\operatorname{Im}\left(\chi_{R}^{(2)}\right)$ spectra of the $\mathrm{H}-\mathrm{O}-\mathrm{H}$ bending mode at the water-air interface. ( $a$ and $b)$ The simulated spectra for the AIMD trajectories at the (a) B3LYP-D3(0) and (b) revPBEO-D3(0) levels of theory. The standard deviations along the amplitude axis were 0.03 and 0.02 for the B3LYP-D3(0) and revPBE0-D3(0) levels of theory, respectively.

evidence for substantial vibrational coupling between bending modes of interfacial water.

SFG spectral calculations using AIMD trajectories at the B3LYP-D3(0) and revPBE0-D3(0) levels of theory confirm this conclusion. The simulated imaginary part spectra of $\chi_{\mathrm{R}}^{(2)}$, $\operatorname{Im}\left(\chi_{\mathrm{R}}^{(2)}\right)$, with various cross-correlation cutoff distances $r_{\mathrm{t}}$ are displayed in Fig. 2. An increase in $r_{\mathrm{t}}$ means that the simulated spectra contain the effects of the intermolecular vibrational coupling between the distant water molecules. These data show that $\operatorname{Im}\left(\chi_{\mathrm{R}}^{(2)}\right)$ is insensitive to the cross-correlation cutoff $r_{\mathrm{t}}$, confirming the notion that the effects of the vibrational coupling on the bending mode spectrum are negligible and thus the SFG lineshape of the water bending mode is not distorted by the isotopic dilution. This ensures that the characteristic frequency of the water bending mode can be obtained with the arbitrary $\mathrm{H}_{2} \mathrm{O}-\mathrm{D}_{2} \mathrm{O}$ concentrations. This is in stark contrast to the $\mathrm{O}-\mathrm{H}$ stretch mode, for which the peak is strongly distorted through the vibrational coupling of the $\mathrm{O}-\mathrm{H}$ stretch modes ${ }^{20,31-33}$ and thus the characteristic frequency of the $\mathrm{O}-\mathrm{H}$ stretch mode can be obtained only for substantial isotopically diluted water (e.g. $5 \% \mathrm{O}-\mathrm{H}$ in $\mathrm{D}_{2} \mathrm{O}$ ).

This finding is important for several aspects. First, since the characteristic frequency of the bending mode can be obtained even from the pure $\mathrm{H}_{2} \mathrm{O}$, we do not have to reduce the bending mode signal further by isotopic dilution to obtain the characteristic frequency. This is a key for probing the SFG bending mode, as the SFG bending mode signal of water is weak and thus the detection of the bending mode signal is often challenging. Second, not only the $\mathrm{H}-\mathrm{O}-\mathrm{H}$ bending mode but also the $\mathrm{H}-\mathrm{O}-\mathrm{D}$ bending mode can be used for probing the interfacial water contribution; because the $\mathrm{H}-\mathrm{O}-\mathrm{D}$ bending mode appears only when investigating the $\mathrm{H}_{2} \mathrm{O}-\mathrm{D}_{2} \mathrm{O}$ mixtures, the $\mathrm{H}-\mathrm{O}-\mathrm{D}$ bending mode can be obtained through the systematic change of the $\mathrm{H}_{2} \mathrm{O}-\mathrm{D}_{2} \mathrm{O}$ mixture composition. If the lineshape is changed through the concentration of $\mathrm{H}-\mathrm{O}-\mathrm{D}$, the $\mathrm{H}-\mathrm{O}-\mathrm{D}$ bending mode contribution is not accessible. Fortunately, since the bending mode SFG lineshape is insensitive to the intermolecular vibrational couplings, one can safely assume that the $\mathrm{H}-\mathrm{O}-\mathrm{D}$ bending lineshape is unchanged. Investigating the $\mathrm{H}-\mathrm{O}-\mathrm{D}$ bending mode is particularly relevant for bio-interfaces, since the $\mathrm{H}-\mathrm{O}-\mathrm{H}$ bending mode overlaps spectrally with the amide mode of proteins. Below, we demonstrate how measuring the water bending mode SFG feature with the tuned $\mathrm{H}_{2} \mathrm{O}-\mathrm{D}_{2} \mathrm{O}$ concentrations can reveal the hydrogen-bonding strength of interfacial water at water- $\mathrm{CaF}_{2}$ and water-protein interfaces.
Enhanced water hydrogen bond strength with increasing $\mathrm{pH}$ at the water- $\mathrm{CaF}_{2}$ interface

Solid-water interfaces affect the electrochemical properties and the wetting properties of materials. The hydroxyl groups chemisorbed onto solid surfaces often complicate the vibrational responses, causing conflicting interpretation of the water $\mathrm{O}-\mathrm{H}$ stretch mode. For example, the $\mathrm{O}-\mathrm{H}$ stretch mode centered at $\sim 3660 \mathrm{~cm}^{-1}$ at the water-silica surface has been attributed to the different molecular origins - the silanol group ${ }^{15}$ and the interfacial water molecules. ${ }^{18,19}$ Similarly, even though the $\mathrm{O}-\mathrm{H}$ stretch mode at $\sim 3650 \mathrm{~cm}^{-1}$ has been assigned to the $\mathrm{Ca}-\mathrm{O}-\mathrm{H}$ group at the water- $\mathrm{CaF}_{2}$ interface, ${ }^{10,11}$ a possible contribution from interfacial water cannot be strictly excluded. Here, by probing the $\mathrm{H}-\mathrm{O}-\mathrm{H}$ bending mode, we quantify the hydrogen bond strength of the interfacial water.

We measured the SFG intensity $\left(I^{(2)}\right)$ spectra for the $\mathrm{H}-\mathrm{O}-\mathrm{H}$ bending mode of the $\mathrm{H}_{2} \mathrm{O}-\mathrm{CaF}_{2}$ interface. These are displayed in Fig. 3(a). The intensity spectra show a peak-like feature at $\mathrm{pH} 2$, while a dip feature was observed around $1650-1750 \mathrm{~cm}^{-1}$ at $\mathrm{pH} 12$ due to the destructive interference between the non-resonant contribution and the bending mode signal. The peak-to-dip transition with $\mathrm{pH}$ increasing from 2 to 12 indicates that the sign of the water bending mode changes. This is attributed to flipping of the water orientation due to the change of the sign of the $\mathrm{CaF}_{2}$ surface charge. Note that the SFG signal at $\mathrm{D}_{2} \mathrm{O}-\mathrm{CaF}_{2}$ does not show any resonant feature. The fit of the intensity spectra (see the ESI $\dagger$ ) indicates that the peak frequency of the $\mathrm{H}-\mathrm{O}-\mathrm{H}$ bending mode band at $\mathrm{pH}=2$ is $1633 \pm 2 \mathrm{~cm}^{-1}$, while it is $1662 \pm 3 \mathrm{~cm}^{-1}$ at $\mathrm{pH}=12$ in the $\operatorname{Im}\left(\chi^{(2)}\right)$ spectra. Since a higher frequency of the bending mode represents a stronger hydrogen bond, ${ }^{24,34,35}$ the variation of the bending mode frequency indicates that the hydrogen bond strength of the interfacial water at the water- $\mathrm{CaF}_{2}$ interface enhances as the $\mathrm{pH}$ increases.

To relate the $\mathrm{H}-\mathrm{O}-\mathrm{H}$ bending mode with the $\mathrm{O}-\mathrm{H}$ stretch mode, we measured the $\mathrm{O}-\mathrm{H}$ stretch modes of $\mathrm{HDO}$ at the isotopically diluted water- $\mathrm{CaF}_{2}$ interface. The spectra are displayed in Fig. 3(b). Note that, to correlate the vibrational coupling-absent $\mathrm{H}-\mathrm{O}-\mathrm{H}$ bending mode spectra with the $\mathrm{O}-\mathrm{H}$ stretch spectra which are largely modulated by the vibrational couplings, ${ }^{20,36,37}$ we used isotopically diluted water for the $\mathrm{O}-\mathrm{H}$
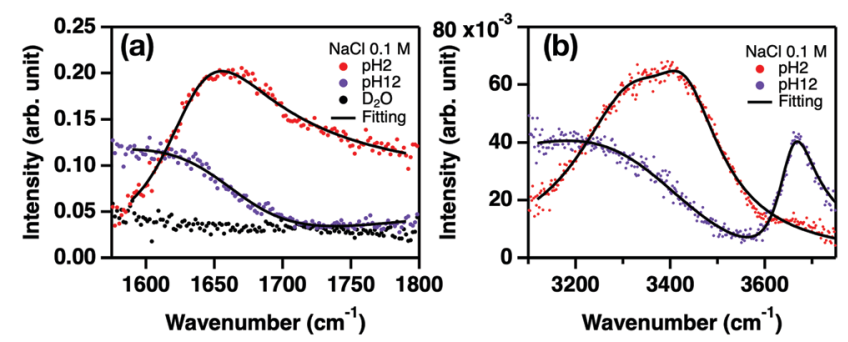

Fig. 3 SFG intensity spectra at the water $-\mathrm{CaF}_{2}$ interface with $\mathrm{pH} 2$ and 12 . (a) $\mathrm{H}-\mathrm{O}-\mathrm{H}$ bending mode spectra for $\mathrm{H}_{2} \mathrm{O}$. (b) O-H stretch mode SFG spectra for the $\mathrm{H}_{2} \mathrm{O}: \mathrm{D}_{2} \mathrm{O}$ mixture $(1: 2$ ratio). The $\mathrm{pH}$ was adjusted using $\mathrm{HCl}$ or $\mathrm{NaOH}$, and the total ion concentration was adjusted to be $0.1 \mathrm{M}$ by adding $\mathrm{NaCl}$ to the solution. 
stretch mode to suppress the vibrational couplings. The following empirical relation between the bending mode and stretch mode frequencies; ${ }^{24,35,38}$

$$
\omega_{\text {str }}^{\mathrm{g}}-\omega_{\text {str }}=a\left(\omega_{\text {bend }}^{\mathrm{g}}-\omega_{\text {bend }}\right)^{2}+b\left(\omega_{\text {bend }}^{\mathrm{g}}-\omega_{\text {bend }}\right),
$$

is employed to decode the $\mathrm{O}-\mathrm{H}$ stretch spectra of water. Here, $a=0.0216$ and $b=-1 / 0.2583 . \omega_{\text {bend }}^{\mathrm{g}}$ and $\omega_{\text {str }}^{\mathrm{g}}$ indicate gas-phase water vibration frequencies of $1590.6 \mathrm{~cm}^{-1}$ and $3706 \mathrm{~cm}^{-1}$, while $\omega_{\text {bend }}^{1}$ and $\omega_{\text {str }}^{1}$ indicate liquid-phase water vibration frequencies of $1650 \mathrm{~cm}^{-1}$ and $3400 \mathrm{~cm}^{-1}$, respectively. ${ }^{36,39}$

Eqn (2) indicates the expected $\mathrm{O}-\mathrm{H}$ stretch peak frequency, concluded from the bend frequency of interfacial water at $\mathrm{pH}=2$, is $3503 \pm 9 \mathrm{~cm}^{-1}$, which agrees with the $\mathrm{O}-\mathrm{H}$ stretch mode frequency obtained from the fit of the intensity spectra of $3426 \pm 3 \mathrm{~cm}^{-1}$. Since the $\mathrm{Ca}-\mathrm{O}-\mathrm{H}$ groups are expected to be absent at $\mathrm{pH} 2,{ }^{10,11}$ the $\mathrm{O}-\mathrm{H}$ stretch signature can be reasonably considered to arise solely from the interfacial water. Because the $\mathrm{O}-\mathrm{H}$ stretch mode is much stronger than the non-resonant contribution unlike the $\mathrm{H}-\mathrm{O}-\mathrm{H}$ bending mode, the nonresonant contribution is nearly zero for the $\mathrm{O}-\mathrm{H}$ stretch spectra, while the non-resonant contribution is certainly large for the $\mathrm{H}-\mathrm{O}-\mathrm{H}$ bending mode spectra (see the ESI $\dagger$ ). Subsequently, we focus on the case of $\mathrm{pH} 12$. The $\mathrm{H}-\mathrm{O}-\mathrm{H}$ bending mode frequency of $1662 \mathrm{~cm}^{-1}$ suggests an expected $\mathrm{O}-\mathrm{H}$ stretch mode frequency of $3320 \pm 12 \mathrm{~cm}^{-1}$. This expected $\mathrm{O}-\mathrm{H}$ stretch frequency is in good agreement with the peak frequency of $3363 \pm 15 \mathrm{~cm}^{-1}$ for the $3000-3500 \mathrm{~cm}^{-1} \mathrm{O}-\mathrm{H}$ stretch band. This suggests that the $3000-3500 \mathrm{~cm}^{-1} \mathrm{O}-\mathrm{H}$ band arises from the interfacial water, not the $\mathrm{Ca}-\mathrm{O}-\mathrm{H}$ groups, consistent with the previous studies. ${ }^{10,11}$ The results also demonstrate that the $3650 \mathrm{~cm}^{-1}$ peak does not originate from water, but indeed from the $\mathrm{Ca}-\mathrm{O}-\mathrm{H}$ groups. As such, the comparison of the O-H stretch SFG spectra with the bending mode spectra can offer an unambiguous assignment of the vibrational signatures at complex aqueous interfaces.

\section{Strong hydrogen bond network of water in the vicinity of HSA protein}

The water-protein interaction is the key for biological function and in particular, protein folding. Although vibrational spectroscopy has been used to understand such interactions, characterizing the hydrogen bond strength of water and protein is usually complicated because the vibrational signal of water is governed by the many water molecules that are not in direct contact with the protein. Meanwhile, the SFG signal originates intrinsically from the interfacial water and proteins at the water-protein interfaces, allowing for a selective probe of the interfacial water structure. Proteins, however, contain the $\mathrm{O}-\mathrm{H}$ groups in their sidechains as well as $\mathrm{N}-\mathrm{H}$ groups in their backbone, both of which can contribute to the vibrational response in $3000 \mathrm{~cm}^{-1}<$ $\omega<3500 \mathrm{~cm}^{-1}$, along with the $\mathrm{O}-\mathrm{H}$ stretch mode of the interfacial water. ${ }^{40}$ Thus, it is prohibited to extract the net water contribution from the $\mathrm{O}-\mathrm{H}$ stretch SFG spectra.

Here, we demonstrate that the water contribution can be extracted from the bending mode SFG spectra. Probing the $\mathrm{H}-\mathrm{O}-\mathrm{H}$ bending mode in the presence of protein is challenging, because the $\mathrm{H}-\mathrm{O}-\mathrm{H}$ bending mode peak $\left(1590-1650 \mathrm{~cm}^{-1}\right)$ is overwhelmed by the strong amide I mode peak $\left(1620-1700 \mathrm{~cm}^{-1}\right)$. To avoid the frequency overlap with the amide I mode, we probe the $\mathrm{H}-\mathrm{O}-\mathrm{D}$ bending mode $\left(1450-1500 \mathrm{~cm}^{-1}\right)$ instead of the $\mathrm{H}-\mathrm{O}-\mathrm{H}$ bending mode. Although the $\mathrm{H}-\mathrm{O}-\mathrm{D}$ bending mode frequency overlaps with the frequency of the amide II mode (1480-1570 $\left.\mathrm{cm}^{-1}\right),{ }^{41}$ the amide II mode signal is small in the SFG spectra, due to the small Raman cross-section of the amide II mode. ${ }^{42}$ We would like to note that, to properly disentangle the $\mathrm{H}-\mathrm{O}-\mathrm{D}$ bending mode contribution from the potentially overlapped amide II mode, and to verify the procedure, one needs to carefully compare the amplitude of the bending mode against the $\mathrm{H}-\mathrm{O}-\mathrm{D}$ concentration.

We measured the SFG intensity $\left(I^{(2)}\right)$ at the isotopically diluted water-air interface in the presence of HSA interfaces by varying the $\mathrm{H}_{2} \mathrm{O} / \mathrm{D}_{2} \mathrm{O}$ concentration. Fig. 4(a) displays the variation of the SFG spectra. In the absence of $\mathrm{HDO}-i . e$. for pure $\mathrm{H}_{2} \mathrm{O}$ and $\mathrm{D}_{2} \mathrm{O}$-, the SFG spectra are indistinguishable and show a signal at $1410 \mathrm{~cm}^{-1}$ and a relatively weak signal between 1500 and $1550 \mathrm{~cm}^{-1}$, attributed to the $\mathrm{H}-\mathrm{C}-\mathrm{H}$ bending mode or the symmetric stretch mode of $\mathrm{R}^{-\mathrm{COO}^{-}}$, and the amide II mode of HSA, respectively. These spectral features closely resemble the SFG spectra obtained at $\mathrm{pH} 6.3$ for the water-air interface with bovine serum albumin. ${ }^{43}$ To extract the $\mathrm{H}-\mathrm{O}-\mathrm{D}$ bending mode contribution from the spectra for the $\mathrm{H}_{2} \mathrm{O} / \mathrm{D}_{2} \mathrm{O}$ mixtures, we fit the spectra (see the ESI $\dagger$ ). The obtained imaginary parts of the response are displayed in Fig. 4(b), with the $\mathrm{H}-\mathrm{O}-\mathrm{D}$ bending contributions highlighted, while the amplitude of the $\mathrm{H}-\mathrm{O}-\mathrm{D}$

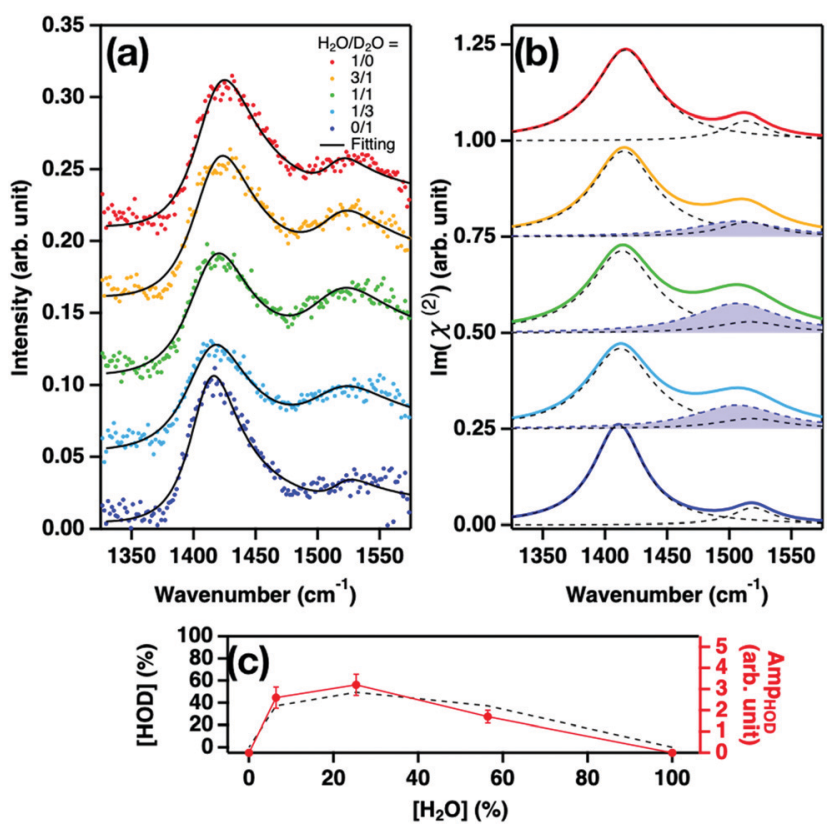

Fig. 4 SFG responses at the water-air interface in the presence of HSA. (a) SFG intensity spectra, $I^{(2)}(\omega)$, of the water-air interface in the presence of $\mathrm{HSA}$ with $1 / 0,3 / 1,1 / 1,1 / 3$, and $0 / 1 \mathrm{H}_{2} \mathrm{O} / \mathrm{D}_{2} \mathrm{O}$ mixtures. The black lines are the fit. The spectra are offset by 0.05 for clarity. (b) Imaginary part spectra of the HSA and $\mathrm{H}-\mathrm{O}-\mathrm{D}$ water contributions obtained from the fits. The $\mathrm{H}-\mathrm{O}-\mathrm{D}$ contribution is highlighted in blue. The spectra are offset by 0.25 for clarity. (c) The amplitude of the $\mathrm{H}-\mathrm{O}-\mathrm{D}$ contribution vs. $\mathrm{H}_{2} \mathrm{O}$ fraction. Theoretical prediction denoted by the black broken line is obtained from eqn (1). 
contribution is plotted in Fig. 4(c). The variation of the amplitude shows excellent agreement with the $\mathrm{H}-\mathrm{O}-\mathrm{D}$ concentration inferred from eqn (1), confirming the peak assignment. The center frequency for the $\mathrm{H}-\mathrm{O}-\mathrm{D}$ contribution is $1506 \pm 3 \mathrm{~cm}^{-1}$. This frequency is substantially higher than the frequency of the $\mathrm{H}-\mathrm{O}-\mathrm{D}$ bending mode in the bulk $\left(\sim 1460 \mathrm{~cm}^{-1}\right),{ }^{44}$ indicating that the interfacial water molecules near the HSA protein are more strongly hydrogen-bonded than water in the bulk.

\section{Discussion}

Above, we have shown that the vibrational couplings and spectral overlap of the $\mathrm{O}-\mathrm{H}$ stretch mode of water with other $\mathrm{O}-\mathrm{H}$ and $\mathrm{N}-\mathrm{H}$ stretch modes prohibited the extraction of the interfacial water contribution from the $\mathrm{O}-\mathrm{H}$ stretch mode SFG spectra, while the SFG probe of the water bending mode allows us to gain the information on the hydrogen bond strength of the interfacial water. The application of the bending mode is not only limited to the water-solid interface or the water-protein interface but also used for studying the interfacial water at the water-lipid/surfactant interfaces, ${ }^{45-47}$ alcohol-water mixture interfaces, ${ }^{48-52}$ and acid aqueous solution interface. ${ }^{53,54}$

For example, 1,2-dipalmitoyl-sn-glycero-3-phospho-(1'-racglycerol) (DPPG) is an essential lipid for a cell membrane, and the DPPG-water interfaces have been studied with $\mathrm{O}-\mathrm{H}$ stretch SFG to understand the water orientations. ${ }^{45-47}$ However, a DPPG lipid has multiple $\mathrm{O}-\mathrm{H}$ groups in its headgroup, prohibiting the extraction of the net interfacial water contribution. Having established here that the characteristic frequency of the bending mode can be obtained without any isotopic dilution, it is apparent from our previous bending SFG data at the water-DPPG interface $^{24}$ that the hydrogen bond strength of water near DPPG is similar to that in the bulk. We observed that the characteristic frequency of the interfacial $\mathrm{H}-\mathrm{O}-\mathrm{H}$ bending mode is $1653 \mathrm{~cm}^{-1}$, i.e., very similar to $1650 \mathrm{~cm}^{-1}$ in the bulk liquid. The conclusion that water at a DPPG interface is hydrogen-bonded with bulk-like strength also affects the interpretation of the vibrational dynamics of the O-H stretch mode near DPPG, reported to be much slower than the bulk water vibrational dynamics. ${ }^{55}$

We point out that the bending mode SFG probe will be powerful for unveiling the interfacial structure of water at the interface of water-alcohol mixtures. This system is important for dilution processes as well as general physical chemistry, because upon mixing with water, the structure of the water and alcohol mixture is drastically changed, resulting in the variation of the vapor pressure. The microscopic structures of the water-alcohol interfaces have been studied with SFG in the O$\mathrm{H}$ stretch region, ${ }^{4-52}$ but the net contribution of the interfacial water has not been accessed. The different contributions to the $\mathrm{O}-\mathrm{H}$ stretch spectrum can now be disentangled by probing the water bending mode in the SFG measurements.

The water bending mode signal is useful not only because its frequency carries information on the hydrogen bond strength of the interfacial water molecules, but also because its amplitude is sensitive to the chemical compositions. Theoretical studies have suggested that, at the water- $\mathrm{TiO}_{2}$ interface, chemisorbed and physisorbed conformations of the interfacial water molecules ${ }^{17}$ are easily distinguished through the bending mode amplitude. ${ }^{56}$ As such, for several cases, the water bending mode can be a very sensitive probe for the microscopic structure of the interfacial water molecules.

In summary, our combined simulations and experiments showed that the SFG feature of the water bending mode of interfacial water at the water-air interface is not distorted upon the isotopic dilution of water (Fig. 1) and thus the vibrational coupling of the bending modes of the interfacial water is virtually absent (Fig. 2). Thus, the characteristic frequency of the bending mode can be obtained for an arbitrary mixture of the $\mathrm{H}_{2} \mathrm{O}-\mathrm{D}_{2} \mathrm{O}$ concentration, unlike the $\mathrm{O}-\mathrm{H}$ stretch mode, where the characteristic frequency can be obtained only for the isotopically diluted water. ${ }^{36}$ Since the use of the isotopic diluted water reduces the SFG signal drastically, our findings ensure that one can use pure $\mathrm{H}_{2} \mathrm{O}$ for gaining the maximum SFG amplitude or can use the $\mathrm{H}-\mathrm{O}-\mathrm{D}$ bending mode for avoiding frequency overlap with the amide I mode. We have applied this new approach for exploring the $\mathrm{CaF}_{2}-$ water interface, and found that an increase in $\mathrm{pH}$ enhances the hydrogen bond strength of interfacial water (Fig. 3). Even for a complex protein-water interface, the bending mode SFG could successfully probe the interfacial water contribution in the $\mathrm{H}-\mathrm{O}-\mathrm{D}$ bending mode region and revealed that the interfacial water near the HSA protein shows a stronger hydrogen bond strength than bulk water (Fig. 4). Altogether, our work manifests that the water bending mode is a promising tool for understanding interfacial water structures at various complex interfaces.

\section{Experimental details}

\section{SFG measurement}

We used a femtosecond Ti:Sapphire amplified laser system (Coherent Libra, $\sim 800 \mathrm{~nm}, \sim 50 \mathrm{fs}, 1 \mathrm{kHz}$ ). The visible and IR beams overlapped spatially and temporally at the sample surface with their incident angles of $64^{\circ}$ and $40^{\circ}$ with respect to the surface normal, respectively. All the spectra were taken in ssp (denoting s-, s-, and p-polarized SFG, visible, and IR, respectively) polarization combination. The signals at the water-air and waterprotein interfaces were normalized to the non-resonant signal taken from the $\mathrm{z}$-cut quartz, while the signals at the water- $\mathrm{CaF}_{2}$ interface were normalized to that from the gold- $-\mathrm{CaF}_{2}$ interface. The details of the experiments can be found in the ESI. $\dagger$

\section{Computational procedure}

We computed the bending mode SFG spectra based on the surface-specific velocity-velocity correlation function formalism; ${ }^{57}$

$$
\begin{aligned}
\chi_{x x z}^{(2), \mathrm{R}}(\omega) \sim & \frac{1}{i \times \omega^{2}} \int_{0}^{\infty} \mathrm{d} t \mathrm{e}^{-i \omega t} \\
& \times\left\langle\sum_{i, j} g_{\mathrm{ds}}\left(z_{i}(0)\right) g_{\mathrm{t}}\left(r_{i j}(0) ; r_{\mathrm{t}}\right) \dot{r}_{z, i}^{\mathrm{OM}}(0) \frac{\dot{\boldsymbol{r}}_{j}^{\mathrm{OM}}(t) \cdot \boldsymbol{r}_{j}^{\mathrm{OM}}(t)}{\left|\boldsymbol{r}_{j}^{\mathrm{OM}}(t)\right|}\right\rangle,
\end{aligned}
$$


where $z_{i}(t)$ is the $z$-coordinate of the $i$ th oxygen atom at time $t$, and $g_{\mathrm{ds}}\left(z_{i}\right)$ is the function for the dividing surface to selectively extract the vibrational responses of $\mathrm{D}_{2} \mathrm{O}$ molecules near the interface. $r_{j}^{\mathrm{OM}}(t)$ is the vector pointing from the oxygen atom $(\mathrm{O})$ to the middle point of the two hydrogen atoms $(\mathrm{M})$ for water molecule $i$ at time $t . g_{\mathrm{t}}\left(r_{i j}(0) ; r_{\mathrm{t}}\right)$ excludes the correlation of the different water molecules, $i$ and $j$, when the oxygen-oxygen distance of water molecules $i$ and $j\left(r_{i j}(0)\right)$ is larger than the cutoff distance $r_{\mathrm{t}}{ }^{58}$ We chose $r_{\mathrm{t}}=0,4$, and $6 \AA$ in Fig. 2 . We used the AIMD trajectories of the $\mathrm{D}_{2} \mathrm{O}$-air interface obtained in our previous work. ${ }^{59}$ In short, we used the B3LYP-D3(0) and revPBE0-D3(0) levels of theory for the AIMD simulations. The $\mathrm{D}-\mathrm{O}-\mathrm{D}$ bend frequencies were converted to the $\mathrm{H}-\mathrm{O}-\mathrm{H}$ bend frequencies by using a factor of $1 / 0.735$. The detail of the scaling factors of the frequency axis can be found in the ESI. $\dagger$

\section{Conflicts of interest}

The authors declare no competing interest.

\section{Acknowledgements}

We thank Prof. Masanari Okuno for fruitful discussion. We acknowledge the financial support from the MaxWater Initiative of the Max Planck Society and the ERC Starting Grant (Grant No. 336679). K. M. acknowledges the DFG for support (DFG project No. 427448850). Open Access funding provided by the Max Planck Society.

\section{Notes and references}

1 O. Björneholm, M. H. Hansen, A. Hodgson, L. M. Liu, D. T. Limmer, A. Michaelides, P. Pedevilla, J. Rossmeisl, H. Shen, G. Tocci, E. Tyrode, M. M. Walz, J. Werner and H. Bluhm, Chem. Rev., 2016, 116, 7698-7726.

2 K. Meister, S. Strazdaite, A. L. DeVries, S. Lotze, L. L. C. Olijve, I. K. Voets and H. J. Bakker, Proc. Natl. Acad. Sci. U. S. A., 2014, 111, 17732-17736.

3 K. Engelhardt, U. Weichsel, E. Kraft, D. Segets, W. Peukert and B. Braunschweig, J. Phys. Chem. B, 2014, 118, 4098-4105.

4 K. Engelhardt, W. Peukert and B. Braunschweig, Curr. Opin. Colloid Interface Sci., 2014, 19, 207-215.

5 R. Pandey, K. Usui, R. A. Livingstone, S. A. Fischer, J. Pfaendtner, E. H. G. Backus, Y. Nagata, J. Fröhlich-nowoisky, L. Schmüser, S. Mauri, J. F. Scheel, D. A. Knopf, U. Pöschl, M. Bonn and T. Weidner, Sci. Adv., 2016, 2, e1501630.

6 D. Nagasawa, T. Azuma, H. Noguchi, K. Uosaki and M. Takai, J. Phys. Chem. C, 2015, 119, 17193-17201.

7 J. Kim and P. S. Cremer, ChemPhysChem, 2001, 2, 543-546. 8 Y. R. Shen and V. Ostroverkhov, Chem. Rev., 2006, 106, 1140-1154.

9 D. Lis, E. H. G. Backus, J. Hunger, S. H. Parekh and M. Bonn, Science, 2014, 344, 1138-1142.

10 R. Khatib, E. H. G. Backus, M. Bonn, M.-J. Perez-Haro, M.-P. Gaigeot and M. Sulpizi, Sci. Rep., 2016, 6, 24287.
11 K. A. Becraft and G. L. Richmond, Langmuir, 2001, 17, 7721-7724.

12 S. Kataoka, M. C. Gurau, F. Albertorio, M. A. Holden, S.-M. Lim, R. D. Yang and P. S. Cremer, Langmuir, 2004, 20, 1662-1666.

13 S. Urashima, A. Myalitsin, S. Nihonyanagi and T. Tahara, J. Phys. Chem. Lett., 2018, 9, 4109-4114.

14 A. Myalitsin, S. H. Urashima, S. Nihonyanagi, S. Yamaguchi and T. Tahara, J. Phys. Chem. C, 2016, 120, 9357-9363.

15 L. Dalstein, E. Potapova and E. Tyrode, Phys. Chem. Chem. Phys., 2017, 19, 10343-10349.

16 L. Zhang, C. Tian, G. A. Waychunas, Y. R. Shen, V. Di and L. Berkeley, J. Am. Chem. Soc., 2008, 7686-7694.

17 S. Hosseinpour, F. Tang, F. Wang, R. A. Livingstone, S. J. Schlegel, T. Ohto, M. Bonn, Y. Nagata and E. H. G. Backus, J. Phys. Chem. Lett., 2017, 8, 2195-2199.

18 O. Isaienko and E. Borguet, Langmuir, 2013, 29, 7885-7895. 19 J. D. Cyran, M. A. Donovan, D. Vollmer, F. S. Brigiano, S. Pezzotti, D. R. Galimberti, M. P. Gaigeot, M. Bonn and E. H. G. Backus, Proc. Natl. Acad. Sci. U. S. A., 2019, 116, 1520-1525.

20 J. Schaefer, E. H. G. Backus, Y. Nagata and M. Bonn, J. Phys. Chem. Lett., 2016, 7, 4591-4595.

21 Y. Tong, T. Kampfrath and R. K. Campen, Phys. Chem. Chem. Phys., 2016, 18, 18424-38430.

22 M. Vinaykin and A. V. Benderskii, J. Phys. Chem. Lett., 2012, 3, 3348-3352.

23 A. Kundu, S. Tanaka, T. Ishiyama, M. Ahmed, K. Inoue, S. Nihonyanagi, H. Sawai, S. Yamaguchi, A. Morita and T. Tahara, J. Phys. Chem. Lett., 2016, 7, 2597-2601.

24 T. Seki, S. Sun, K. Zhong, C. C. Yu, K. Machel, L. B. Dreier, E. H. G. Backus, M. Bonn and Y. Nagata, J. Phys. Chem. Lett., 2019, 10, 6936-6941.

25 C. Dutta and A. V. Benderskii, J. Phys. Chem. Lett., 2017, 8, 801-804.

26 Y. Nagata, C.-S. Hsieh, T. Hasegawa, J. Voll, E. H. G. Backus and M. Bonn, J. Phys. Chem. Lett., 2013, 4, 1872-1877.

27 D. R. Moberg, S. C. Straight and F. Paesani, J. Phys. Chem. B, 2018, 122, 4356-4365.

28 J. Meija, Z. Mester and A. D'Ulivo, J. Am. Soc. Mass Spectrom, 2006, 17, 1028-1036.

29 M. Wolfsberg, A. A. Massa and J. W. Pyper, J. Chem. Phys., 1970, 53, 3138-3146.

30 J. C. Duplan, L. Mahi and J. L. Brunet, Chem. Phys. Lett., 2005, 413, 400-403.

31 P. A. Pieniazek, C. J. Tainter and J. L. Skinner, J. Chem. Phys., 2011, 135, 044701.

32 C.-S. Tian and Y. R. Shen, J. Am. Chem. Soc., 2009, 131, 2790-2791.

33 S. Nihonyanagi, T. Ishiyama, T.-K. Lee, S. Yamaguchi, M. Bonn, A. Morita and T. Tahara, J. Am. Chem. Soc., 2011, 133, 16875-16880.

34 S. Imoto, S. S. Xantheas and S. Saito, J. Chem. Phys., 2013, 139, 044503.

35 Y. Ni and J. L. Skinner, J. Chem. Phys., 2015, 143, 014502. 
36 F. Perakis, L. De Marco, A. Shalit, F. Tang, Z. R. Kann, T. D. Kühne, R. Torre, M. Bonn and Y. Nagata, Chem. Rev., 2016, 116, 7590-7607.

37 M. Sovago, R. K. Campen, G. W. H. Wurpel, M. Müller, H. J. Bakker and M. Bonn, Phys. Rev. Lett., 2008, 100, 173901.

38 M. Falk, Spectrochim. Acta, Part A, 1984, 40, 43-48.

39 Y. Maréchal, J. Mol. Struct., 1994, 322, 105-111.

40 K. Meister, A. Paananen and H. J. Bakker, Phys. Chem. Chem. Phys., 2017, 19, 10804-10807.

41 J. Kong and S. Yu, Acta Biochim. Biophys. Sin., 2007, 39, 549-559.

42 D. Kurouski, R. P. Van Duyne and I. K. Lednev, Analyst, 2015, 140, 4967-4980.

43 K. Engelhardt, A. Rumpel, J. Walter, J. Dombrowski, U. Kulozik, B. Braunschweig and W. Peukert, Langmuir, 2012, 28, 7780-7787.

44 M. Falk, J. Raman Spectrosc., 1990, 21, 563-567.

45 J. A. Mondal, S. Nihonyanagi, S. Yamaguchi and T. Tahara, J. Am. Chem. Soc., 2012, 134, 7842-7850.

46 X. Chen, W. Hua, Z. Huang and H. C. Allen, J. Am. Chem. Soc., 2010, 132, 11336-11342.

47 L. B. Dreier, Y. Nagata, H. Lutz, G. Gonella, J. Hunger, E. H. G. Backus and M. Bonn, Sci. Adv., 2018, 4, eaap7415.
48 J. Sung, K. Park and D. Kim, J. Phys. Chem. B, 2005, 109, 18507-18514.

49 G. Ma and H. C. Allen, J. Phys. Chem. B, 2003, 107, 6343-6349.

50 X. Li, J. Liu, K. Lin, Y. Zhang, Y. Zhang, R. Zheng, Q. Shi, Y. Guo and Z. Lu, J. Phys. Chem. C, 2019, 123, 12975-12983.

51 T. Ishihara, T. Ishiyama and A. Morita, J. Phys. Chem. C, 2015, 119, 9879-9889.

52 H. Chen, W. Gan, B. H. Wu, D. Wu, Y. Guo and H. F. Wang, J. Phys. Chem. B, 2005, 109, 8053-8063.

53 S. Gopalakrishnan, D. Liu, H. C. Allen, M. Kuo and M. J. Shultz, Chem. Rev., 2006, 106, 1155-1175.

54 C. M. Johnson, E. Tyrode, A. Kumpulainen and C. Leygraf, J. Phys. Chem. C, 2009, 113, 13209-13218.

55 P. C. Singh, K. I. Inoue, S. Nihonyanagi, S. Yamaguchi and T. Tahara, Angew. Chem., Int. Ed., 2016, 55, 10621-10625.

56 M. F. Calegari Andrade, H. Y. Ko, R. Car and A. Selloni, J. Phys. Chem. Lett., 2018, 9, 6716-6721.

57 T. Ohto, K. Usui, T. Hasegawa, M. Bonn and Y. Nagata, J. Chem. Phys., 2015, 143, 124702.

58 Y. Nagata and S. Mukamel, J. Am. Chem. Soc., 2010, 132, 6434-6442.

59 T. Ohto, M. Dodia, J. Xu, S. Imoto, F. Tang, F. Zysk, T. D. Kühne, Y. Shigeta, M. Bonn, X. Wu and Y. Nagata, J. Phys. Chem. Lett., 2019, 10, 4914-4919. 